\title{
Nuclear astrophysics in our time: supernovae, neutron stars and binary neutron star mergers
}

\author{
Debades Bandyopadhyay ${ }^{\mathrm{a}}$ \\ Saha Institute of Nuclear Physics, HBNI, 1/AF Bidhannagar, Kolkata 700064, India \\ Published online 17 May 2021 \\ (C) EDP Sciences, Springer-Verlag GmbH Germany, part of Springer Nature 2021
}

\begin{abstract}
This volume focuses on topical issues in nuclear astrophysics, particularly supernovae, neutron stars, and binary neutron star mergers. An overview of those topics is presented in this editorial. The lasting impact of the Saha equation on astrophysics over the past hundred years is highlighted here.
\end{abstract}

The year 2020 and the beginning of a new decade would be remembered forever in the annals of human history! Who could imagine that a virus would play havoc with human lives and livelihoods in 2020? Of course, this is nothing new. Mankind had experienced this kind of pandemic earlier too and the last one was the Spanish flu of 1918-1920. Though life stands still in a pandemic, science can not! This is amply evident from the race to find a vaccine for COVID-19.

When the pandemic of the Spanish flu loomed large on the world, Meghnad Saha, a young scientist from the University of Calcutta, developed the thermal ionizing theory for unlocking the mysteries of stellar spectra and published those findings in a series of papers with the first one in 1920 [1]. It heralded a new era of Astrophysics - a quantum jump from astronomical studies of the Sun and stars! This gives us immense pleasure to edit this special issue in the centenary year of the Saha thermal ionization equation.

Understanding the final journey of a massive star, after its nuclear fuel has been exhausted, is a fascinating problem because it involves all the forces of nature. The outcome of it may be a supernova explosion and the residue either be a neutron star or a black hole. This is a highly interdisciplinary area of research involving astrophysics, general relativity, nuclear physics and particle physics. The core collapse supernova (CCSN) explosion mechanism is being investigated over the past five decades or so. Still, the detailed theory of successful supernova explosions is eluding us and a challenging problem [2]. The equation of state (EoS) of matter from low to high density and neutrino interactions with matter are important microphysics inputs in CCSN $[3,4]$.

The spectacular discovery of the first binary neutron star (BNS) merger event GW170817 in gravitational waves and across the electromagnetic spectrum resulted in a wealth of information about the short gamma ray burst, binary chirp mass, tidal deforma-

a e-mail: debades.bandyopadhyay@saha.ac.in (corresponding author) bility, speed of gravitational waves, and Hubble constant [5]. Furthermore, it is manifestly evident that GW170817 was a huge boost for nuclear astrophysics communities around the world as dense matter EoS in neutron stars and heavy element synthesis through rprocess nucleosynthesis in the ejected neutron-rich matter could be probed.

The birth of neutron stars through supernova explosion mechanism and binary neutron star mergers are delicately linked by various aspects of nuclear astrophysics. It is the linkage that we want to explore in this issue of the European Physical Journal (EPJ) on Special Topics. A brief summary of all scholarly articles in this special issue is presented in the following paragraphs.

The thermal ionization equation developed by Meghnad Saha in 1920 was a pathbreaking work in the field of Astrophysics [1]. It was hypothesised in 1917 by Arthur S. Eddington that atoms in the Sun were in a highly ionized state. Meghnad Saha demonstrated this using the thermal ionization equation along with Bohr's atomic model for the first time in 1920. The Saha equation was extremely fruitful in explaining the spectral sequence of stars in terms of physical parameters such as temperature and pressure. Even after one hundred years, the Saha equation is widely applied in the EoS of supernova matter, big bang nucleosynthesis, r-process nucleosynthesis, and synthesis of light elements in relativistic heavy-ion collisions. The first article of Gangopadhyay in this special issue focuses on the historical account of the Saha equation and its applications cutting across different fields of physics [6].

The detection of neutrinos from SN1987A was a major success of the CCSN explosion mechanism. Neutrinos are trapped in the Fe core during the gravitational core collapse when the density reaches $\sim 10^{12}$ $\mathrm{g} / \mathrm{cm}^{3}$ and are emitted after the supernova explosion. Nineteen neutrino events of SN1987A were recorded over 11 s by water Cerenkov detectors at KamiokandeII, Japan and the Irvine-Michigan-Brookhaven facility 
in the USA. Energies of detected neutrinos were a few tens of $\mathrm{MeV}$ and compatible with the prediction of the CCSN theory. Now many such neutrino facilities are operational to detect neutrinos from the next galactic CCSN. Bhattacharjee and Kar review a novel mechanism to explore the CCSN and properties of neutrinos [7]. It is discussed in their article that neutrinos from the CCSN might excite nuclei of detector materials and produce neutrons due to charged current interactions of electron type (anti)neutrinos as well as neutral current interactions of all flavours of (anti)neutrinos with detector nuclei. Those neutrons could shed light on the CCSN mechanism. It is also highlighted that simultaneous observations of a CCSN in $\mathrm{Pb}$ and $\mathrm{Fe}$ detector would provide information about the fractions of $\mu$ - and $\tau$-type neutrinos in the CCSN neutrino flux.

Neutron star crust and its EoS up to the saturation density from below are very well constrained by experimental nuclear physics inputs obtained by studying finite nuclei. On the other hand, the EoS at supranuclear densities and large neutron-proton asymmetries could be probed by masses, radii, and tidal deformabilities of neutron stars. The EoS encodes the information of nuclear matter parameters that constitute infinite nuclear matter. Nuclear matter parameters may be correlated to each other as well as nuclear and neutron star observables. Thus these correlations would lead to a better understanding of the nuclear matter EoS. In the next article, Agrawal, and collaborators review correlations of nuclear matter parameters with various properties of finite nuclei and neutron stars within mean-field frameworks [8].

The detection of the gravitational wave signal from the first BNS merger GW170817 by the LIGO/VIRGO collaboration opened a new window in the study of neutron stars. The tidal deformation of a neutron star due to the other in the binary is encoded in the phase evolution of the gravitational wave signal during the late stage inspiral. The analysis of the gravitational wave signal in GW170817 led to the determination of the tidal deformability parameter. It is well known that the tidal deformability parameter is sensitive to the EoS. The tidal deformability parameter extracted from the gravitational wave signal of GW170817 estimated the neutron star radius imposing an important constraint on the EoS of dense matter. On the other hand, the gravitational mass of the hot merger remnant in GW170817 was known very accurately though there was no detection of post merger gravitational waves in the range of a few $\mathrm{kHz}$ due to the lack of sensitivity of gravitational wave detectors. However, it was possible to determine an upper bound on the maximum mass of a neutron star assuming the remnant was short-lived and collapsed to a black hole. This also imposed another strong constraint on the EoS [9-11]. All these demonstrate that the EoS of neutron star matter might be probed from low to high temperatures in BNS mergers.

A very pertinent question is what neutron stars are made of. Various novel phases of dense matter are conjectured to exist in neutron star interior. Exotic phases of matter such as hyperons, Bose-Einstein condensate of antikaons, and quarks might appear in neutron star matter. There might be the imprint of such matter on gravitational wave signals in BNS mergers. The next three articles look into those aspects of dense matter.

In one of those articles, Hanauske et al. highlight different scenarios of hadron-quark phase transition (HQPT) in the differentially rotating merger remnant - prompt phase transition (PPT) and delayed phase transition (DPT) [12]. In the PPT case immediately after the merger, a stable quark matter core is formed in the merger remnant. However, the HQPT in DPT might occur in the hypermassive neutron star (HMNS) in the late post merger phase and result in a metastable hypermassive hybrid star (HMHS). The dynamics of HMHS in DPT is studied at length using the BNS merger simulation in numerical relativity in this article. Large macroscopic oscillations of the maximum density are found in the late stage post merger evolution of HMHS. It is predicted that the imprint of HQPT could be detected in future gravitational wave observations of BNS mergers by analysing the gravitational wave emission from a differentially rotating HMHS.

In another article, Nandi and Pal investigate the appearance of an exotic form of matter such as hyperons and quarks in neutron star interior in light of GW170817 [13]. Baryons are treated within the relativistic mean-field approach, whereas the bag model is adopted for quarks. It is noted that hyperons appear in neutron stars having masses heavier than 1.4 solar mass. Consequently, a faster decrease of tidal deformability with increasing mass is found in hyperon stars than in nucleons-only stars. On the other hand, the phase transition from nuclear to quark matter might occur in lower mass stars depending on the threshold condition for the appearance of quark matter as determined by bag model parameters. Several EoSs with quarks are found to be consistent with the tidal deformability bound extracted for a $1.4 \mathrm{M}_{\odot}$ neutron star from the gravitational wave signal of GW170817.

Equations of State of matter for a wide range of values for baryon density, temperatures and positive charge fractions are essential ingredients in CCSN and BNS merger simulations in numerical relativity. A large pool of EoSs involving nucleons-only matter, hyperon matter, and quark matter which are compatible with $\geq 2 \mathrm{M}_{\odot}$ neutron stars, are available for simulations. Malik et al. present the results of a new EoS table involving the Bose-Einstein condensate of $K^{-}$mesons as well as thermally produced (anti)kaons [14]. The main finding of this work is the softening of the EoS due to the appearance of the antikaon condensate at higher densities and the dissolution of the condensate and large populations of thermal (anti)kaons at higher temperatures. This new EoS table is available for the study of CCSN and BNS merger simulations and the imprint of the Bose-Einstein condensate of $\mathrm{K}^{-}$mesons in gravitational wave signals by the scientific community.

The presence of superfluidity in neutron star matter is another interesting possibility. It is inferred that pulsar glitches, sudden spin-up in neutron stars, are 
the manifestation of superfluid neutron matter in the crust [15]. On the other hand, the rapid cooling of the neutron star in the supernova remnant Cassiopeia A is attributed to the outcome of neutron superfluidity in the core $[16,17]$. The pairing in neutron matter is fundamental to the study of neutron superfluidity. Ramanan and Urban review s- and p-wave pairing in pure neutron matter [18]. It is noted that the behaviour of the gap for $s$-wave spin-singlet $\left({ }^{1} S_{0}\right)$ pairing with density is consistent with the results of quantum Monte Carlo and many-body calculations. However, uncertainties arise in the gap at higher densities due to the effective neutron mass, screening, and short range correlations. The $p$ wave spin-triplet $\left({ }^{3} P_{2}-{ }^{3} F_{2}\right)$ pairing might be possible in neutron star core. The $p$-wave pairing gaps in pure neutron matter suffer from large uncertainties. The realistic case of neutrons admixed with protons in the dense core might improve this situation.

It has been long argued that strange baryons might appear at 2-3 times the saturation density in neutron stars [19]. Hyperons are found to impact the EoS and maximum neutron star mass. The appearance of hyperons in dense matter sensitively depends on the interactions between nucleons and hyperons. However, such interactions are not known very accurately due to the lack of sufficient experimental information except $\Lambda$-hypernuclei. Meher and Raha explore the $\Xi^{-}$-nn three-body problem in the maximal spin-isospin channel adopting a low energy pionless halo effective field theory (EFT) at the leading order [20]. The pionless halo EFT provides an alternative assessment about the viability of $\Xi^{-}$-nn system as a three-body bound system using low energy universality. Authors obtain the three-body scattering length in the range $2.6-2.9 \mathrm{fm}$ using three-body binding energies $\sim 3-4 \mathrm{MeV}$ estimated from Faddeev based potential models, as inputs. Consequently, these might imply important features of a possible halo-bound $\Xi^{-}$-nn system.

Nuclear Astrophysics research has entered into an exciting phase with the detection of gravitational waves from binary neutron mergers. We were lucky to observe the first BNS merger in gravitational waves and light because it happened at the right distance. This culminated in a new era of multi-messenger astrophysics involving gravitational waves. Articles of this special issue introduce readers to the interdisciplinary areas of nuclear physics, particle physics, neutron stars, and CCSN, gravitational waves, and numerical relativity. We are hopeful that this volume would be an useful reference for researchers in this field.

Finally, I should acknowledge the help of those without which the compilation of this special issue would not be possible. I am thankful to the Editorial board particularly B. Ananthanarayan for his encouragement to bring out a special issue on topical issues in nuclear astrophysics. It is my pleasure to thank all authors who contribute to this special issue even during this trying time. Last but not least, I am grateful to Sandrine Karpe and Sabine Lehr for patiently guiding me through the editorial process.

\section{References}

1. M.N. Saha, Philos. Mag. 40, 472 (1920)

2. A. Burrows, D. Vartanyan, (n.d.)

3. H.A. Bethe, G.E. Brown, J. Applegate, J.M. Lattimer, Nucl. Phys. A 324, 487 (1979)

4. E. O'Connor, C.D. Ott, Astrophys. J. 730, 70 (2011)

5. B.P. Abbott et al., Phys. Rev. Lett. 119, 161101 (2017)

6. G. Gangopadhyay, Hundred years of the Saha equation and astrophysics. Eur. Phys. J. Spec. Top. (2021). https://doi.org/10.1140/epjs/s11734-021-00005-3

7. P. Bhattacharjee, K. Kar, Supernova neutrino detection through neutron emission by nuclei. Eur. Phys. J. Spec. Top. (2021).https://doi.org/10.1140/epjs/ s11734-021-00002-6

8. B. Agrawal, T. Malik, J.N. De, S.K. Samaddar, Constraining nuclear matter parameters from correlation systematics: a mean-field perspective. Eur. Phys. J. Spec. Top. (2021). https://doi.org/10.1140/epjs/ s11734-021-00001-7

9. L. Rezzolla, E.R. Moist, L.R. Weith, Astrophys. J. Lett. 852, L25 (2018)

10. B. Margalit, B.D. Metzger, Astrophys. J. Lett. 850, L19 (2017)

11. M. Ruiz, S.L. Shapiro, A. Tsokaros, Phys. Rev. D 97, 021501 (2018)

12. M. Hanauske, L. R. Weih, H. Stöcker, L. Rezzolla, Metastable hypermassive hybrid stars as neutron-star merger remnants. Eur. Phys. J. Spec. Top. (2021). https://doi.org/10.1140/epjs/s11734-021-00003-5

13. R. Nandi, S. Pal, Finding quark content of neutron stars in light of GW170817. Eur. Phys. J. Spec. Top. (2021). https://doi.org/10.1140/epjs/s11734-021-00004-4

14. T. Malik, S. Banik, D. Bandyopadhyay, New equation of state involving Bose - Einstein condensate of antikaon for supernova and neutron star merger simulations. Eur. Phys. J. Spec. Top. (2021). https://doi.org/10.1140/ epjs/s11734-021-00006-2

15. G. Baym, C.J. Pethick, D. Pines, M. Ruderman, Nature 224, 872 (1969)

16. C.O. Heinke, W.C.G. Ho, Astrophys. J. Lett. 719, L167 (2010)

17. D. Page, M. Prakash, J.M. Lattimer, A.W. Steiner, Phys. Rev. Lett. 106, 081101 (2011)

18. S. Ramanan, M. Urban, Pairing in pure neutron matter. Eur. Phys. J. Spec. Top. (2021). https://doi.org/10. 1140/epjs/s11734-021-00008-0

19. N.K. Glendenning, Phys. Rev. D 46, 1274 (1992)

20. G. Meher, U. Raha, Investigation of $X i^{-} n n(S=-2)$ hypernucleus in low-energy pionless halo effective theory. Eur. Phys. J. Spec. Top. (2021). https://doi.org/ 10.1140/epjs/s11734-021-00007-1 